\title{
Presentación Dossier: Abordajes metodológicos en torno a las movilidades en la Región Metropolitana de Buenos Aires
}

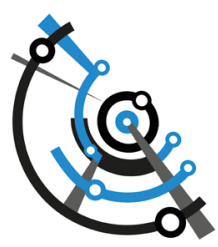

Q Maximiliano Augusto Velázquez

Centro de Estudios de Transporte Área Metropolitana, Instituto Superior de Urbanismo, Universidad de Buenos Aires. https://orcid.org/oooo-0001-7271-0288

\author{
Verónica Pérez \\ Consejo Nacional de Investigaciones Científicas y Técnicas. Instituto de Altos Estudios \\ Sociales. Universidad Nacional de San Martín. https://orcid.org/oooo-0003-3307-0489
}

La convocatoria a construir un dossier sobre movilidades en la región metropolitana de Buenos Aires nos exigió, primeramente, instalar la mirada en una perspectiva históricopolítica que nos permita devolver al concepto de movilidad una significación situada ¿De qué hablamos cuando hablamos de movilidades hoy y específicamente, cuando la anclamos en un territorio tan complejo y heterogéneo como es el de la Región Metropolitana de Buenos Aires? ¿Es posible abordar la problemática de la movilidad desde una abstracto sin tiempo ni lugar? ¿Qué procesos intervienen en la configuración de las múltiples movilidades? ¿Cómo se producen hoy las movilidades en el territorio y qué implicancias tiene esta diversidad de procesos sobre la delimitación del concepto? ¿Mediante qué instrumentos teórico-metodológicos podemos abordar su estudio?

Siguiendo a Tim Cresswell (2008) y otros, las movilidades son prácticas sociales con sentido, por lo tanto, experiencias que son representadas y producen cultura, así como expresan y producen relaciones sociales y de poder. Los objetos de estudio de la movilidad suelen ser híbridos, con fuertes componentes multidisciplinares, espacializados, mediando prácticas humanas con elementos no humanos como los vehículos, las redes, las normas y las infraestructuras. La movilidad emerge así como un complejo proceso sociotecnológico que tiene necesariamente especificidad territorial.

El universo de los objetos que se mueven abarca -al menos- tres dimensiones de la realidad, sin duda interrelacionadas. Cosas, personas e ideas establecen relaciones entre sí en un tiempo y espacio determinado, configurando una totalidad de relaciones que, en su mismo devenir, van dotando de diversas significaciones tanto a la experiencia del moverse como a los espacios en los cuales se producen los movimientos. Los territorios en los cuales las movilidades se producen también van siendo afectados morfológica y sensorialmente, a medida que estas se desarrollan.

En esta dirección, pensar la región metropolitana hoy, implica tener presente la gran transformación de la que ha sido objeto, atravesada por procesos de carácter global y local, entre los cuales el desarrollo de las distintas movilidades ha sido uno de los principales elementos estructurantes de su actual morfología. En ella, en la Gran 
Ciudad, es posible identificar movimientos de personas y bienes, de capitales de distinto origen y destino, movimientos de ideas en general y específicamente acerca de aquello que las ciudades deberían ser o expresar. Es posible también, registrar ritmos e intensidades en todas estas maneras de producirse la movilidad, e inclusive inmovilidades y detenciones.

\section{El movimiento de personas en la metrópolis}

Abarcar en algún aspecto específico un territorio como el de la Región Metropolitana de Buenos Aires (RMBA), cuya extensión supera los $18.380 \mathrm{~km} 2$ distribuidos en 44 unidades administrativas -definidas utilizando un criterio funcional basado en el movimiento diario que realizan las personas en su vida cotidiana-, resulta de por sí un desafío. Con más de 14 millones de habitantes, sus funciones de producción generan más del $50 \%$ del PBI que produce nuestro país. Ello exige reflexionar respecto de las diferentes escalas de la movilidad -global, regional o local-, con sus diversos gradientes, así como sus localizaciones de núcleos problemáticos, demandas sociales e infraestructuras.

En el caso particular de las movilidades de personas que se producen en la región, los datos disponibles ${ }^{1}$ permiten observar cambios significativos en la matriz de viajes durante los últimos 40 o 50 años. Los viajes dentro de la Ciudad Autónoma de Buenos Aires (CABA) se redujeron significativamente entre 1970 y 2010, pasando de representar del 43,3\% al 24,6\% del total; misma tendencia mostraron los viajes entre CABA y la Provincia de Buenos Aires (PBA), que disminuyeron en 7.5 puntos (del 21,6\% pasaron al 14,1\%); mientras que los que tienen lugar al interior de la PBA, se incrementaron significativamente (del 35,1\% del total de viajes en 1970, en 2010 representaban el $61,3 \%$ ). Por su parte, la movilidad de cercanías (es decir, la producida al interior de cada localidad) alcanzó, según ENMODO 2010, el 35,3\% del total de los viajes; y si el agregado fueran los partidos y las comunas, alcanzaron el 54,6\%.

La modernidad incorporó cambios en los flujos cotidianos acompañados por el crecimiento extensivo de la mancha urbana, plasmados en infraestructuras predominantemente viales. Sin duda que la gran transformación se visualiza en el fuerte incremento del uso del automóvil, que pasó de representar el 16,7\% de los viajes diarios en 1970, al $23,7 \%$ en 2010 y su contracara, la progresiva disminución en el uso de modos masivos que cayeron de $72,5 \%$ a $64,5 \%$ en igual período. El análisis del reparto modal indica una caída en las preferencias por los servicios de transporte colectivo masivo, en particular, del autotransporte colectivo de pasajeros. El reparto modal a favor del automóvil ha aumentado en línea con el aumento de 9\% de hogares que poseen un automóvil entre 1970 y 2010. Por su parte, el uso del ferrocarril y del subterráneo se mantuvo relativamente estable en términos relativos.

Estas transformaciones, con sus consecuencias negativas sobre el medio ambiente y la utilización inequitativa del espacio vial, fueron a su vez consecuencia y causa eficiente en la nueva configuración urbana que se caracterizó, al igual que la mayoría de las ciudades de la globalización, (Prevot S., 2002; Sassen, 2007, Ciccolella, 2014) por fuertes contrastes riqueza-probreza, espacios fragmentados y discontinuos y profundas brechas de hipermovilidad e inmovilidad entre sus habitantes. 
Transformaciones urbanas que afectaron las condiciones de vida, incluidas las posibilidades y condiciones de movilidad de los grupos humanos y que a su vez fueron el substrato principal en la configuración de múltiples y divergentes experiencias de la ciudad y de la auto representación de los grupos sociales.

En tiempos recientes, la mirada urbana se orientó a escala mundo, proponiendo ciudades en donde se recupere la "escala humana", redirigiendo las políticas sectoriales hacia formulaciones como las de movilidad sustentable, con promoción de la movilidad activa del caminar y la bicicleta. También adquirió relevancia la promoción del uso del transporte masivo y público, con el objeto de mitigar el impacto de la movilidad privada, tanto en términos energéticos como ambientales, inscribiendo incipientemente políticas de descarbonización en el transporte urbano. No obstante, esas nuevas lógicas impuestas discursivamente aún no han desplazado las miradas tradicionales del transporte en la región.

Repensar la movilidad en la región metropolitana desde una perspectiva sociocultural y a raíz de los cambios mencionados, permite reconocer cuatro dimensiones de análisis entrelazadas e interrelacionadas:

" La presencia de una reconfiguración de las infraestructuras de transporte que en su relación con la urbanización, transita -en lo morfológico- desde el modelo de suburbanización tentacular expansivo al de modalidades insulares. Este enfoque cuestiona la noción de centro-periferia y el pensamiento respecto de la primacía de la radialidad de los corredores de transporte, redirigiendo la mirada hacia una ciudad policéntrica con múltiples jerarquías.

"La persistencia de la vigencia de cuestiones socio-territoriales no resueltas y agravadas, tales como la inequidad urbana y la fragmentación territorial, resultantes del desarrollo económico-espacial desigual de larga data. El barrio y lo local exigen reflexiones específicas para los tipos de movilidad tradicionales como el trabajo y la educación, así como la predominancia de situaciones de movilidades de cercanía, en donde lo peatonal, los viajes cortos, parecen ser la media al moverse dentro de territorios contenidos.

" Unos procesos de larga duración de accesibilidad diferenciada, acentuada en varias localidades de la metrópolis, que entran en disputa con la deseable universalización del derecho a la ciudad, a vivir y desarrollarse como individuo en la sociedad metropolitana. Destaca el enfoque de la movilidad justa o el derecho a la movilidad, en el marco de una articulación conflictiva entre jurisdicciones y agencias gubernamentales.

» La dinámica de movilidad urbana, la conectividad espacial y la accesibilidad social se erigen como factores prevalentes de segregación y polarización social, en donde, particularmente el acceso al transporte público, no compensa las inequidades económicas, sociales y culturales de las diversas localidades de la metrópolis.

Observamos a estas cuatro dimensiones entrecruzadas por las teorías de la movilidad que lentamente van incluyendo y reformulando los enfoques clásicos disciplinares del transporte (Velázquez y Dmuchowsky, 2015), particularmente los relativos a la ingeniería, la planificación y el urbanismo. Los modelos de planificación de transporte tradicionales se resquebrajan, carentes de datos de calidad para calibrarlos y ausentes de análisis socioculturales.

Por lo tanto, estamos en presencia de un marco teórico-conceptual que se encuentra aún en formación, más parecido a una constelación de teorías y abordajes que han acercado autores, tanto clásicos como contemporáneos, de las ciencias sociales (sociología, geografía, antropología y urbanismo) articulando con los saberes de las ciencias 
aplicadas (ingeniería y arquitectura), en un intercambio multi e interdisciplinar (Kruger, Orduna y Velázquez, 2017)

En este enjambre de problemas emergen nuestros interrogantes, ¿cómo abordar tanta diversidad? ¿Mediante qué herramientas conceptuales y metodológicas? ¿Qué potencialidad nos brindan los instrumentos existentes? ¿Contamos con suficientes fuentes de información para abordar y caracterizar los cambios que se van produciendo? ¿Esas fuentes son accesibles para la sociedad y para los investigadores? ¿Qué nuevas tecnologías pueden interpelar la movilidad cotidiana tanto de personas como de bienes o información? ¿Mediante qué herramientas podemos intervenir planificadamente en la transformación positiva y progresiva de un espacio socio-territorial tan complejo como la RMBA?

Los trabajos que conforman este Dossier, constituyen un importante aporte para seguir profundizando el conocimiento de los desafíos que nos presentan los interrogantes planteados.

Ramiro Segura y Mariana Chavez desarrollan una estrategia metodológica para el análisis antropológico de la experiencia metropolitana en el Corredor Sur de la RMBA. Su preocupación por comprender la experiencia metropolitana los conduce a utilizar los "relatos de espacio" como principal operador metodológico, enfatizando su cualidad como producciones narrativas en las que se intersectan formas de hacer y formas de creer, informando simultáneamente sobre las prácticas en la metrópoli y las forma de significar la vida en ella. A partir de un riguroso trabajo de construcción de la muestra, arriban a la conformación de seis tipos socio-urbanos sobre los cuales implementan un total de 90 entrevistas. La estrategia elegida les permite abordar tres cuestiones generales: el "contenido" de los relatos de espacio les facilita comprender dinámicas clave de la movilidad cotidiana; la "forma" de los relatos de espacio, les posibilita identificar y abordar dimensiones y sentidos claves de la experiencia metropolitana y la inclusión de distintas localidades del corredor sur de la RMBA, les permite caracterizar experiencias metropolitanas moduladas por el lugar de residencia, entre otras variables.

El artículo de Laura Aón y su equipo propone poner en valor las potencialidades de los conceptos e instrumentos clásicos reformulándolos y combinándolos, con el objetivo de desarrollar nuevas formas (más ágiles, flexibles, extensivas y sobre todo más económicas para los gobiernos) de construir información de movilidad cuantitativa y cualitativa, tomando como unidad de análisis territorial al concepto de "atractor de viaje" y aplicándolo a investigaciones específicas en el ámbito de la salud y la educación en el área del Gran La Plata. Se proponen encuestas en actividades no-residenciales, es decir actividades que implican importantes flujos de movilidad urbana, como la educación, el trabajo y la salud, como una estrategia de investigación relevante para el estudio de los patrones de movilidad, según grupos socio económicos de la población, lugares de residencia y tamaño y estructura de los hogares, desde un camino inverso a las encuestas domiciliarias. Esto es, basados en considerar a los diversos espacios de atracción de viajes como representativos de la matriz productiva a escala urbana.

El trabajo de Daniela Soldano propone el estudio de los viajes metropolitanos ligados al consumo, como un potente operador metodológico para el estudio de la cuestión urbana y específicamente, de la experiencia de la desigualdad. El recorte espacio temporal se sitúa en el partido de José C. Paz, entre los años 2003 y 2014. El diseño metodológico cualitativo basado en el método etnográfico, centrado en la producción entrevistas en profundidad, observaciones, historias de vida e historias de casos de familias, le permite un acercamiento a la experiencia de la desigualdad en ese territorio. Entre los hallazgos, cabe resaltar su señalamiento acerca de que el consumo produce subjetividades marcadas por el capital espacial. 
El aporte de Natalia Verónica Neri, Carla Galeota y Lucila Capelli retoma metodologías clásicas en el abordaje de la movilidad urbana, como son las generadas por las Encuestas de Movilidad Domiciliarias. Su trabajo indaga sobre la accesibilidad en el Área Metropolitana de Buenos Aires (AMBA) a centros educativos por parte de los niños, niñas y adolescentes (NNA). Entre los hallazgos de la explotación de datos de la encuesta destaca la evidencia de patrones diferenciados según el territorio y el grupo social, y la importancia de los viajes no motorizados para el desarrollo de estas movilidades. En tono prospectivo, estas constataciones les permiten reforzar la importancia de la formulación de políticas públicas que, por ejemplo, propongan mejorar las condiciones de caminabilidad barrial en torno a escuelas.

Finalmente, el trabajo de Candela Hernández sitúa la mirada en los usuarios del sistema de transporte público colectivo de la RMBA, a partir de la pregunta por los mecanismos existentes para la tramitación de las disconformidades que emergen de las experiencias de viaje. Aquí, el operador metodológico es el análisis de los diseños institucionales y su eficacia como mecanismos para canalizar dichas disconformidades, tanto desde la perspectiva de los usuarios del sistema, como de quienes tienen a su cargo la elaboración de políticas. En sus reflexiones finales, Hernández alerta sobre la brecha existente entre gestión y demandas de los pasajeros, así como también, sorbe las asimetrías en los tratamientos de la información recabada por distintos órganos. Esto último no es menor, en tanto se erige en una importante limitante a la construcción de datos consolidados que permitan construir conocimiento para la toma de decisiones. En esta dirección, concluye que la indefensión de los usuarios del transporte público no se circunscribe sólo a cuestiones de prestación. 


\section{Q Bibliografía}

"Ciccolella, P. (2014). Metrópolis Latinoamericanas: más allá de la globalización. Buenos Aires: Café de las Ciudades.

"Cresswell, T. (2008). Constellations of mobility. Egham, UK: Institute of English Studies, University of London.

» Krüger, R. Orduna, M. y Velázquez M. (2017). La interdisciplinariedad en la planificación de transporte, en Müller et alt (compiladores), Transporte Urbano e Interurbano en la Argentina. Aportes desde la investigación (pp. 15-24), Buenos Aires: EUDEBA.

» Ministerio de Economía (1973). Estudio Preliminar del Transporte de la Región Metropolitana (EPTRM). Tomo 1. Buenos Aires: Secretaría de Estado de Transporte y Obras Públicas.

»Prevot Schapira, M. F. (2002). Buenos Aires en los años 90: metropolización y desigualdades. Eure 28, (85), 31-50.

"Sassen, S. (2007). Una sociología de la globalización. Buenos Aires: Akal.

"Secretaría de Transportes de la Nación (2010). Encuesta de Movilidad Domiciliaria del AMBA (ENMODO). 2010.

"Velázquez, M. y Dmuchowsky, J. (2015). Movilidades polarizadas. Nuevos aportes teóricos para indagar la fragmentación y segregación metropolitana. En S. Vidal-Koppmann, S. (Comp.). Metrópolis en Mutación (pp.101-128). Buenos Aires: Café de las Ciudades.

\section{Maximiliano Augusto Velázquez / maxovelazquez@gmail.com}

Es Magíster en Planificación Urbano y Regional y licenciado en Sociología de Universidad de Buenos Aires (UBA); docente de grado y posgrado en UBA; investigador Programa Interdisciplinario de la Universidad de Buenos Aires sobre Transporte (PIUBAT) y Centro de Estudios de Transporte Área Metropolitana (CETAM) del Instituto Superior de Urbanismo (ISU) de UBA.

\section{Verónica Pérez / veronicaperez@gmail.com}

Es Doctora en Ciencias Sociales y Licenciada en Sociología (UBA). Investigadora Adjunta del CONICET (IDAES-UNSAM). Profesora de grado y posgrado (FSOCUBA). Coordinadora del Programa Interdisciplinario de Estudios del Transporte y la Movilidad (PIETyM-IDAES-UNSAM). Integrante del Programa Interdisciplinario de la UBA sobre Transporte (PIUBAT). 